\title{
Surface characterization of plasma treated meso-carbons
}

\section{Kunio Esumi, Masaaki Sugiura, Toshiaki Mori, Kenjiro Meguro and Hidemasa Honda}

(Received 27 May. 1986)

\author{
Department of Applied Chemistry and Institute of Colloid and \\ Interface Science, Science University of Tokyo, \\ Kagurazaka, Shinjuku-ku, Tokyo 162, Japan
}

\begin{abstract}
A surface treatment of meso-carbons was conducted by using plasma of oxygen, nitrogen, and ammonia and was characterized by means of FTIR-PAS, FTIR-ATR, zeta potential, and heat of immersion techniques. From FTIR-PAS and FTIR-ATR measurements, it was found that the carboxyl groups were formed by the oxygen plasma, while the amino groups were produced by ammonia and nitrogen plasma. The isoelectric point of the meso-carbons treated by the oxygen plasma was shifted to lower value, but that by the ammonia and nitrogen plasma to higher value. Further, the heat of immersion in water for the plasma treated meso-carbons increased due to formaion of hydrophilic groups.
\end{abstract}

KEYWORDS: meso-carbon, plasma, surface treatment

\section{Introduction}

It is known (1) that a mesophase transformation takes place in graphitizable organic materials such as pitches during pyrolysis at temperature between 350 and $500^{\circ} \mathrm{C}$. During the heat treatment of pitches, mesophase spherules are formed due to an accumulation of oriented polycondensed aromatic hydrocarbons. The mesophase spherules that are separated as quinoline insolubles from the mesophase pitches are generally called meso-carbon microbeads. When these spherules meet each other, coalescence occurs to produce larger droplets, leading eventually to bulk mesophase.

The surface treatment of these meso-carbons by plasma has been conducted to modify their surface properties. The present authors have reported $(2,3)$ that the turbidity of plasma treated meso-carbons in aqueous solution is enhanced, indicating the improvement of wettability of the plasma treated meso-carbons. However, surface characterization of plasma treated meso-carbons is not fully understood.

In this work the surfaces of plasma treated meso-carbons were characterized more in detail by means of FTIR-ATR,-PAS, heat of immersion, and zeta potential techniques.

\section{Experimental}

Materials. Bulk meso-carbons were prepared from coal-tar pitch and petroleum pitch by heattreatment for a few hours at about $400^{\circ} \mathrm{C}$. The petroleum pitch used in this study was obtained from FCC decant oil. Thus obtained bulk mesocarbons were crushed and passed through a 200 Tyler mesh. Here, the bulk meso-carbons prepared from coal-tar and petroleum one are referred to $\mathrm{CBM}$ and $\mathrm{PBM}$.

Procedure. About 3 grams of these samples were charged into a reaction vessel, followed by degassing down to 1.5 Torr. Each gas was then passed at a rate of $100 \mathrm{ml} / \mathrm{min}$ into the vessel while evacuated samples were treated using glow discharges generated by a radio frequency of 13.57 $\mathrm{MHz}$ under various high-frequency powers and treatment times. The pressure during the treatment was kept about 3 Torr.

Measurements. The zeta-potential of the suspension containing treated meso-carbons was determined by an Electrophoresis apparatus 
(Laser Zee Model 500, Pen Kem).

Known amounts of these samples were put into a glass-ample tube and degassed at $1 \times 10^{-3}$ Torr for 1 hour. Then, the glass-ample tube was sealed. The heat of immersion in water for these samples was measured by a calorimeter (Tokyo Riko Co., Ltd.) at $25^{\circ} \mathrm{C}$.

The surface of samples treated with the plasma was measured by FTIR-PAS (EPA-84) and FTIR-ATR(DR-81) (Japan Spectroscopic Co., Ltd).

\section{Results and Discussion}

When the meso-carbons were treated with respective gas plasma under conditions such as high-frequency powers $(25,50 \mathrm{~W})$, changes in weight of the meso-carbons were observed with the treating time. By the oxygen plasma, the
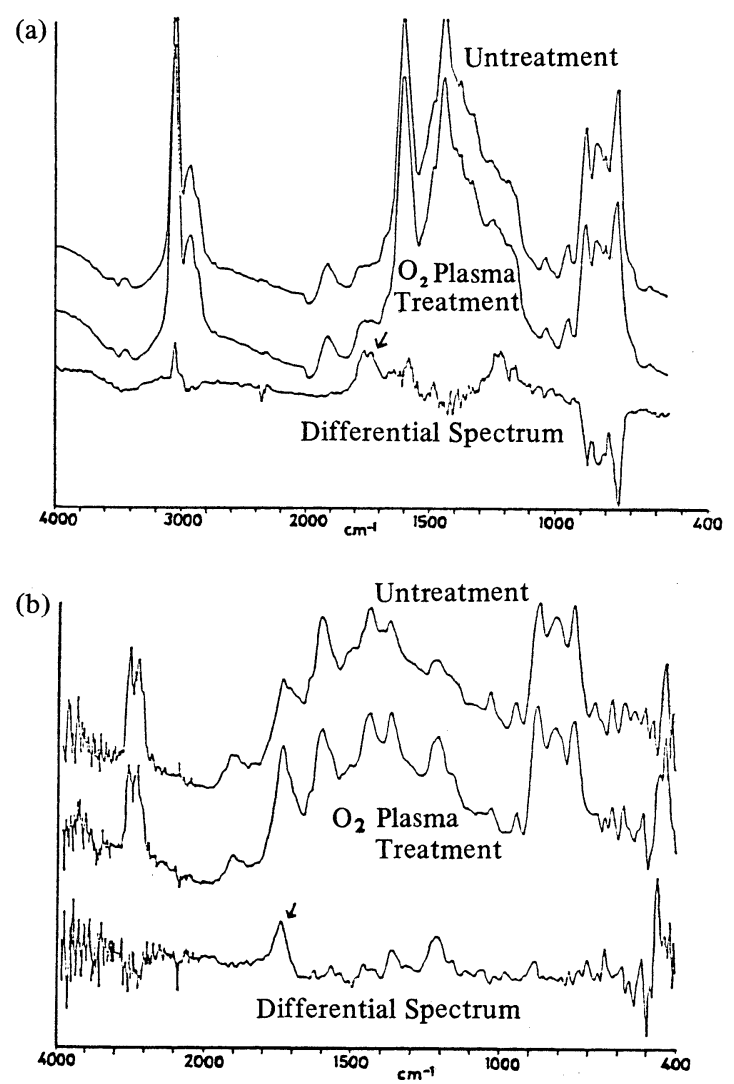

Fig. 1 (a) FTIR-ATR spectra of untreated and oxygen plasma treatment of CBM, (b) FTIR-PAS spectra of untreated and oxygen plasma treatment of PBM. weight of the meso-carbons decreased remarkably with an increase in the treating time and in the high-frequency power for both CBM and PBM; about $10 \mathrm{wt} \%$ loss for the former and about 20 wt $\%$ loss for the latter at $3 \mathrm{hr}$. On the other hand,both nitrogen and ammonia plasma treatment increased slightly the weight of both CBM and PBM.

The surface analysis of the meso-carbons treated by the respective plasmas was carried out by FTIR-ATR and FTIR-PAS. Fig.1(a) shows the FTIR-ATR spectra of untreated and oxygen plasma treated CBM (50W, $3 \mathrm{hr}$ ), where in the differential spectrum the absorption band at $1740 \mathrm{~cm}^{-1}$ is observed which is assigned to $\mathrm{C}=\mathrm{O}$ stretching vibration of carboxyl group. Further, the differential band at $700-900 \mathrm{~cm}^{-1}$ is appeared which is assigned to aromatic $\mathrm{C}-\mathrm{H}$ out of plane vibration. The intensity of this band is reduced by the oxygen plasma, indicating that the aromatic $\mathrm{C}-\mathrm{H}$ band of CBM is broken by the plasma. Similar spectra of PBM (50W, $3 \mathrm{hr}$ ) as that of CBM are obtained, for which the result is shown in Fig.1(b); in the differential spectrum the absorption band at $1740 \mathrm{~cm}^{-1}$ is appeared, but the aromatic $\mathrm{C}-\mathrm{H}$ band at $700-900 \mathrm{~cm}^{-1}$ is not observed. This result indicates that compared with CBM, the aromatic $\mathrm{C}-\mathrm{H}$ band of PBM is not susceptible to the attack of the plasma.

Fig. 2 shows the FTIR-ATR spectra of untreated and ammonia plasma treated CBM. From the differential spectrum in Fig.2, the absorption bands at $3400,3050,2920$, and $700-900 \mathrm{~cm}^{-1}$

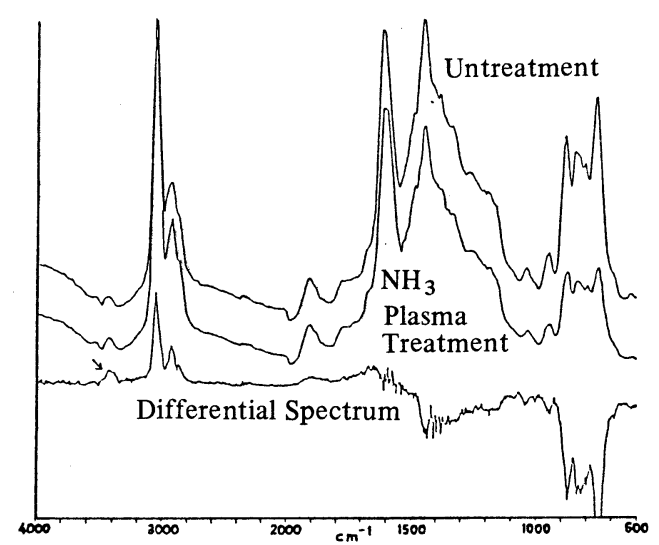

Fig. 2 FTIR-ATR spectra of untreated and ammonia plasma treatment of CBM. 
are observed, which are assigned to $\mathrm{NH}_{2}$ scissoring vibration, aromatic $\mathrm{C}-\mathrm{H}$ stretching vibration, aliphatic, $\mathrm{C}-\mathrm{H}$ stretching vibration, and aromatic $\mathrm{C}-\mathrm{H}$ out of plane vibration, respectively. This figure indicates that the ammonia plasma forms amino groups and breaks the aromatic $\mathrm{C}-\mathrm{H}$ bond of CBM.

The zeta potential of thus treated mesocarbons was measured as a function of suspension's pH. This result is shown in Fig.3. In
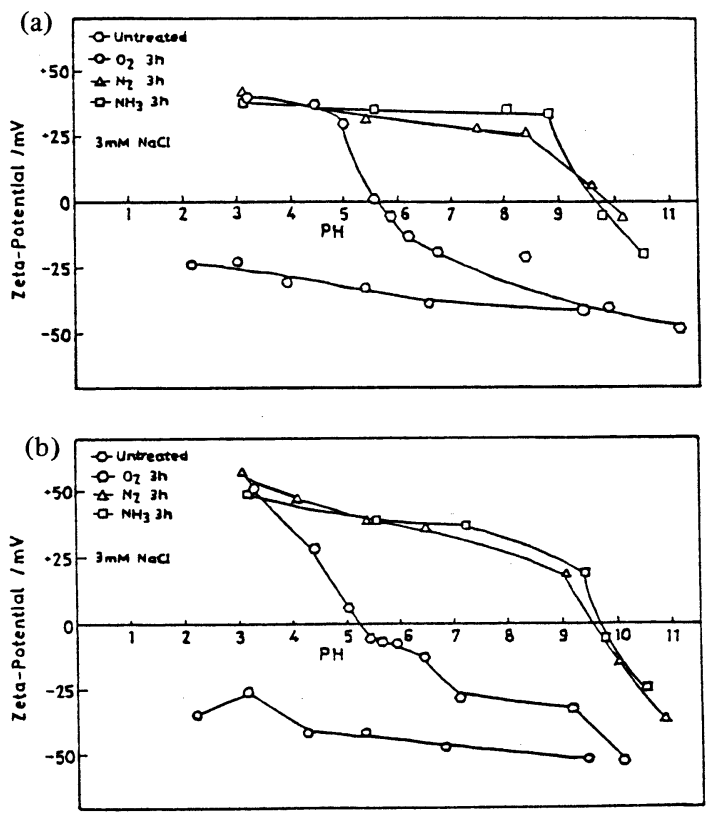

Fig. 3 Zeta potential of $\operatorname{CBM}(a)$ and $\operatorname{PBM}(b)$ treated with respective gas plasma as a function of $\mathrm{pH}$.

both PBM and CBM, the isoelectric points of untreated samples are about 5.2-5.5. When the meso-carbons are treated by the oxygen plasma, the isoelectric point decreases and is below 2, while the ammonia and nitrogen plasmas render the isoelectric point of both CBM and PBM higher value, i-e., 9.5-9.9. These results demonstrate that the oxygen plasma treatment modifies the meso-carbons more acidic surface, while the ammonia and nitrogen one to more basic surface.

Table 1 shows the heat of immersion in water for the plasma treated meso-carbons. After the plasma treatment, the heat of immersion in-
Table 1 Heat of immersion of CMB(a) and PBM(b) treated with respective gas plasma in water.

(a)

\begin{tabular}{cccc}
\hline \multicolumn{4}{c}{ Heat of immersion at $25^{\circ} \mathrm{C}(\mathrm{J} / \mathrm{g})$} \\
Gas & $\mathrm{O}_{2}$ & $\mathrm{~N}_{2}$ & $\mathrm{NH}_{3}$ \\
Time & $50 \mathrm{~W}$ & $50 \mathrm{~W}$ & $50 \mathrm{~W}$ \\
\hline $0 \mathrm{~h}$ & 0.10 & 0.10 & 0.10 \\
$1 \mathrm{~h}$ & 0.18 & 0.36 & 0.45 \\
$2 \mathrm{~h}$ & 1.36 & 1.53 & 0.56 \\
$3 \mathrm{~h}$ & 2.03 & 2.38 & 1.09 \\
$4 \mathrm{~h}$ & -- & 2.26 & 1.00 \\
\hline
\end{tabular}

(b)

Heat of immersion at $25^{\circ} \mathrm{C}(\mathrm{J} / \mathrm{g})$

\begin{tabular}{cccc}
$\begin{array}{c}\text { Gas } \\
\text { Time }\end{array}$ & $\begin{array}{c}\mathrm{O}_{2} \\
50 \mathrm{~W}\end{array}$ & $\begin{array}{c}\mathrm{N}_{2} \\
50 \mathrm{~W}\end{array}$ & $\begin{array}{c}\mathrm{NH}_{3} \\
50 \mathrm{~W}\end{array}$ \\
\hline Oh & 0.09 & 0.09 & 0.09 \\
$1 \mathrm{~h}$ & 0.75 & 0.42 & 0.24 \\
$2 \mathrm{~h}$ & 0.83 & 0.49 & 0.31 \\
$3 \mathrm{~h}$ & 1.35 & 1.34 & 2.36 \\
$4 \mathrm{~h}$ & -- & 0.82 & 0.56 \\
\hline
\end{tabular}

creases with an increase of treating time and reaches a maximum at $3 \mathrm{hr}$. This behavior is in fair agreement with the turbidity behavior (3), suggesting that the meso-carbons become wettable in water due to surface functional groups formed by the plasma treatment and dispersion stability in water is enhanced by inoized functional groups on the meso-carbons.

\section{Acknowledgment}

The authors are indebted to Japan Spectroscopic Co., Ltd for obtaining the FTIR spectra.

\section{References}

1) H.Honda, Mol.Cryst.Liq.Cryst. 94, 97 (1983).

2) M.Sugiura, K.Esumi, K.Meguro, and H.Honda, Bull. Chem. Soc. Jpn. 58, 2638 (1985)

3) K.Esumi, M.Sugiura, T.Mori, K.Meguro, and H.Honda, TANSO, 1986 [No. 125] 57. 